$\Delta$ Palabras clave/ Conjuntos habitacionales arquitectura moderna, patrimonio industrial tipología habitacional.

$\Delta$ Keywords/ Housing complexes, modern architecture, industrial heritage, housing typology

$\Delta$ Recepción/ 18 abril 2017

$\Delta$ Aceptación/ 21 julio 2017

\title{
Arquitecturas de la
} industrialización nacional. El conjunto Papeles Bío-Bío como recinto autónomo primario (1953 hasta la actualidad) ${ }^{1}$.

\author{
National industrialization architectures. \\ The Bío-Bío Papers Complex as a primary \\ autonomous precinct (1953 to date) ${ }^{1}$.
}

\begin{abstract}
Leonel Pérez-Bustamante
Arquitecto, Universidad del Bío-Bío, Chile. Doctor en Urbanismo, Universitat Politécnica de Catalunya, España.

Profesor Asociado, Departamento de Urbanismo, Facultad de Arquitectura, Urbanismo y Geografía Universidad de Concepción, Chile. leperez@udec.cl
\end{abstract}

Pablo Fuentes-Hernández

Arquitecto, Universidad del Bío-Bío, Chile. Doctor en Arquitectura, Universidad Politécnica de Madrid, España.

Profesor Asociado, Departamento de Diseño y Teoría de la Arquitectura, Facultad de Arquitectura, Construcción y Diseño, Universidad del Bío-Bío, Chile. pfuentes@ubiobio.cl

RESUMEN/ A mediados del siglo XX, cuando el proceso de industrialización se desencadenó en Chile, una serie de industrias transcendentales para el desarrollo se ubicó en la ribera del río Biobío, en lo que sería un emplazamiento estratégico. Varias de estas empresas asociaron la instalación fabril con colectivos habitacionales para sus trabajadores. El presente trabajo toma como caso de estudio el conjunto Papeles Bío-Bío, emplazado en la actual comuna de San Pedro de la Paz del Gran Concepción, Chile. Su objetivo es relevar la morfología arquitectónico-urbana del conjunto industrial del caso presentado. Se desarrolla un estudio histórico que releva información a partir de la revisión de fuentes secundarias para, posteriormente, enfocarse en la descripción y análisis de su configuración urbana y arquitectónica, profundizando en sus valores espaciales. ABSTRACT/ In the mid-20 century -which marked the beginning of the industrialization process in Chile- several firms critical for development established themselves on the banks of the Biobio River, in what would later become a strategic location. Many of these companies connected the factory facilities with housing complexes for their workers. This work takes as case study the Bío-Bío Papers complex, located in the current commune of San Pedro de la Paz, Greater Concepción, Chile. Its objective is to highlight the architectural-urban morphology of the case's industrial complex. A historical study is conducted which collects information from secondary sources to then focus on the description and analysis of its

urban and architectural setup, further delving into its spatial values.

\section{INTRODUCCIÓN.}

\section{Estrategias privadas de la industria} papelera en los territorios del río Biobío y la franja costera del Gran

\section{Concepción. La valoración del espacio}

del modelo fordista chileno ha sido escasa especialmente la vivienda y los conjuntos asociados a las fábricas, los que han sido frecuentemente olvidados en los registros sobre patrimonio industrial. Monsálvez, Jaña y Ruiz (2014) enfatizan en la influencia norteamericana en la creación de la Compañía de Acero del Pacífico (CAP), que cambió definitivamente el paisaje urbano del Gran Concepción, aspecto que también ha sido destacado por Brito y Ganter (2014) citando el emblemático caso del histórico barrio Higueras. En Tierra del Fuego, Domínguez (2011) estudió el campamento petrolero Cerro Sombrero, construido por la Empresa Nacional de Petróleo (ENAP), como uno de los proyectos de arquitectura y urbanismo moderno más relevantes edificados en Chile. Acevedo, Ciselli y Rojas (2016) compararon el Campamento Central creado por la empresa Yacimientos Petrolíferos Fiscales (YPF) en Comodoro Rivadavia (Chubut, Argentina), con el
Campamento Cerro Sombrero emplazado en Tierra del Fuego (Chile), indagando en su valor patrimonial.

Recuperar el patrimonio industrial en el sur de Chile cobra relevancia, ya que su preservación interesa al desarrollo y la identidad regional. La fábrica Papeles BíoBío se ubica cercana a la desembocadura del río Biobío en el Océano Pacífico, en la actual comuna de San Pedro de la Paz de Gran Concepción (imagen 1). La empresa inicia sus operaciones en 1957 (Papeles Bío-Bío 2016), en el contexto del proceso de industrialización nacional motivado, entre 
otras cosas, por las necesidades impuestas por el entorno político y económico de posguerra. A mediados del siglo XX, el modelo político de modernización nacional proponía urgentemente intensificar e incrementar la actividad industrial como pilar fundamental del progreso económico y social.

En la elección de su emplazamiento confluyeron como factores desencadenantes dos sistemas territoriales de alcance regiona por entonces en formación, catapultados por esfuerzos coordinados entre el Estado y el sector privado. Por una parte, en sentido este-oeste, el río Biobío constituyó un recurso indispensable para la actividad industrial y actuó como aglutinador de una serie de industrias estatales, privadas y mixtas, que resultaban transcendentales para el desarrollo nacional. Por otra parte, en sentido norte-sur, el sistema urbano costero del Gran Concepción, que en ese momento sostenía un proceso inicial de conurbación y metropolización, se ofreció como lugar privilegiado para la instalación fabril mediante la localización de cordones industriales en lugares privilegiados, aprovechando la existencia de recursos naturales y la actividad industrial decimonónica, que resultaron fundamentales para su desarrollo. Por parte del Estado, de forma directa o indirecta, la Corporación de Fomento (CORFO), impulsó diversos proyectos nacionales de inversión privada, ligados a múltiples sectores de la industria y variados actores económicos. CORFO intentaba apoyar la industria privada otorgando créditos a bajo coste y subvenciones, en un intento por consolidar las políticas de sustitución de importaciones, especialmente para los bienes de capital, a partir de la década de 1940.
Es por ello que se instalaron en torno a las orillas del río Biobío, con especial intensidad en su desembocadura, diversas industrias características del desarrollo industrial regional (Aliste, Contreras y Sandoval 2012; Fuentes 2006). Entre ellas, podemos mencionar la Población Obrera CaupolicánChiguayante (1946), la Villa Presidente Ríos (1949) de la Compañía de Acero del Pacífico (CAP) (Echeñique y Rodríguez 1990); la Población Papeles Bío-Bío (1955) de la Compañía de Papeles y Cartones (CMPC); y la Villa Spring Hill (1971) de la Empresa Nacional del Petróleo (ENAP). Varias de ellas asociaron la instalación fabril con colectivos habitacionales para sus trabajadores.

Desde el sector privado, en tanto industria propia del modelo de industrialización por sustitución de importaciones afianzado en Chile, el rol de CMPC resultó central en las transformaciones ocurridas en este territorio. A comienzos de la década de 1950, la CMPC tomó la decisión de construir una fábrica de papel y otra de celulosa en el sur de Chile, iniciando la búsqueda de terrenos para las plantas industriales en ubicaciones estratégicas en San Pedro, sector entonces perteneciente a la comuna de Coronel, emplazado en la mitad de la franja industrial costera del Gran Concepción; y en Laja, aprovechando la confluencia de los ríos Laja y Biobío y líneas férreas fundamentales para el transporte, además de la presencia de recursos vitales como el agua y los bosques. En las dos décadas durante las que Jorge Alessandri estuvo al mando de la empresa, iniciadas en 1938 (Alessandri, 1969), CMPC ya había comenzado asumir el liderazgo de un proceso de modernización industrial, específicamente en el rubro forestal: “... ya en 1940, La Papelera había dado un

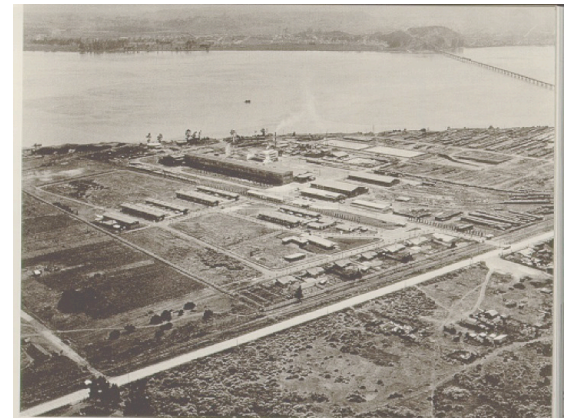

Imagen 1. Vista sur del complejo industrial hacia 1957 (fuente: Archivo Fábrica Papeles Bio-Bio s/f).

paso importante al adquirir el predio Los Pinares, cerca de Concepción, con una de las reservas más importantes de pino insigne de la región..." (Blog El Paleta 2005). Esta es otra de las razones que explica la decisión de proporcionar viviendas para los trabajadores.

También destaca como factor coadyuvante la primera propuesta de planificación metropolitana en el Gran Concepción, que consolida estos procesos de industrialización y transformaciones urbanas. El Plan Regulador Intercomunal de Concepción (PRIC) de 1963 fue desarrollado en el contexto de la planificación urbana con iniciativa estatal en Chile durante las décadas de 1950 y 1960, período durante el cual las ciudades estuvieron sometidas a tensiones que iban desde el crecimiento demográfico hasta el déficit habitacional y el consecuente colapso de sus estructuras urbanas tradicionales (Pérez y Fuentes 2010). Fue un instrumento en el cual el sector industrial era el rubro que caracterizaba la zona con sus correspondientes consecuencias sobre el 
total del área: "En tres lustros apenas la industria se ha convertido en un poderoso foco de atracción de población y sus efectos se han hecho sentir en todos los aspectos de la vida urbana, tendiendo a modernizarla, pero creando a su vez una carga no prevista en todas las funciones urbanas: vivienda, educación, salud, urbanizaciones, transporte de productos elaborados y materias primas" (Burotto 1968: 70).

Se promovieron, entonces, corredores industriales paralelos al litoral, con la incorporación de suelos, a pesar del costo de implementación de infraestructuras. El plan propuso áreas de localización de medianas y grandes industrias. Se trató de lograr una concentración industrial descentralizada dentro de la intercomuna para aprovechar las ventajas de cada área, pues, de ese modo, se pretendía reducir los desplazamientos obreros entre centros urbanos y la agrupación de industrias medianas y pequeñas en complejos que se pudieran nutrir de economías externas. En otro orden de cosas, para una comprensión del lugar físico de implantación de la fábrica de Papeles Bío-Bío y del conjunto habitacional, es necesario referirse a las definiciones de enclave y recinto, que reflejan con nitidez las estrategias territorial y urbana, respectivamente, seguidas por la CMPC en el territorio fluvial y costero del Gran Concepción La definición más elemental de enclave, aportada por la RAE (2018a), es la de "Territorio incluido en otro con diferentes características políticas, administrativas, geográficas, etc.". En efecto, el emplazamiento de Papeles Bío-Bío, del mismo modo que el recinto FACELA en Laja, es una porción de territorio administrado por la empresa, y que se encuentra rodeado y enclavado dentro de otro, en este caso del territorio comunal de San Pedro en el Gran Concepción. Aquí el enclave es privado y discreto en su presencia urbana, y la ciudad apenas se entera de su funcionamiento, tanto antes como ahora, ya que la empresa necesita esa moderación y autonomía para el funcionamiento de la fábrica y del conjunto habitacional. El enclave es la estrategia territorial preferida por la empresa CMPC en sus diferentes locaciones en Puente Alto, San Pedro de Coronel, Laja y Valdivia.

En tanto, el recinto se instituye como una nueva unidad urbana en estos territorios, tanto por la oportunidad como por sus características espaciales. Según la RAE, el término proviene del latín re- 're-' y cinctus 'cercado, rodeado', y se define como "Espacio, generalmente cerrado, comprendido dentro de ciertos límites" (RAE 2018b). Si bien el origen de su utilización como ámbito espacial resulta difícil de precisar, se puede constatar que fue una estrategia de implantación urbana preferente por parte de empresas y servicios del Estado como la Empresa Nacional de Electricidad Sociedad Anónima (ENDESA) y el Ministerio de Obras Públicas (MOP) en la segunda mitad del siglo XX. En los territorios del Gran Concepción y el río Biobío, los conjuntos promovidos por CAP (empresa mixta), ENAP (estatal) o Papelera INFORSA (mixta), despliegan una acción constructora de ciudad que tendió a la producción de tejidos urbanos abiertos. Sin embargo, la CMPC adopta una estrategia industrial que contiene el proyecto residencial como parte del ciclo productivo, ocupándose de construir un conjunto cerrado de notorio control socioespacial que, como se analiza a continuación, incluyó tipos arquitectónicos y vigilancia social, incorporando regulaciones sobre la privacidad.

\section{El enclave y el recinto son entonces}

estrategias espaciales similares a dos escalas diferentes -territorial y urbana-, pero que operan con una misma lógica de partida: la de imprimir el aislamiento espacial y la distancia social con sus entornos, generando segregación respecto de la ciudad y/o sus tejidos urbanos. Enclave y recinto han sido herramientas operativas que, derivadas de los supuestos y fundamentos racionales imperantes a mediados del siglo XX y condensadas en el marco de una ideología desarrollista, posibilitaron la materialización de alternativas de progreso industrial y habitacional en territorios locales. Tradicionalmente, la producción de las periferias metropolitanas en Latinoamérica se ha caracterizado por el desarrollo de enclaves urbanos, residenciales y comerciales (Capron y Esquivel 2016). En clave contemporánea, estos siguen abarcando grandes conjuntos urbanos de vivienda de interés social y conjuntos privados orientados a poblaciones de altos ingresos, entre otros.

Metodológicamente, se desarrolló un estudio de caso que, mediante un proceso cualitativo, analizó una unidad para explorar sus valores patrimoniales tanto urbanos como arquitectónicos. Se examinó aquí un fragmento urbano que permite abordar la revalorización de aquellos conjuntos residenciales asociados a la industria. Un estudio histórico que levanta información a partir de la revisión de fuentes secundarias, da paso a la descripción y análisis pormenorizado de su configuración urbana y arquitectónica. Finalmente, la interpretación de resultados y observación directa sobre equipamientos y tipos arquitectónicos del conjunto habitacional, permitió profundizar en sus

\section{EL CONJUNTO. El 'recinto' como} alternativa de organización funcional.

El conjunto Papeles Bío-Bío es un emprendimiento de una empresa privada que fue concebido como un recinto de autonomía limitada, definida espacial y funcionalmente respecto de la industria y, en consecuencia, del tejido urbano. Se trata de un conjunto relativamente modesto en su superficie, aunque con infraestructura básica suficiente como para caracterizar un barrio Sus límites actuales son el río Biobío por el norte; la línea férrea por el sur y el antiguo camino a Coronel -hoy Avenida Pedro Aguirre Cerda-; terrenos de Ferrocarriles del Estado por el este; y sector Huertos familiares de San Pedro por el oeste. 


\section{CONJUNTO PAPELES BIO-BIO-SAN PEDRO DE LA PAZ}

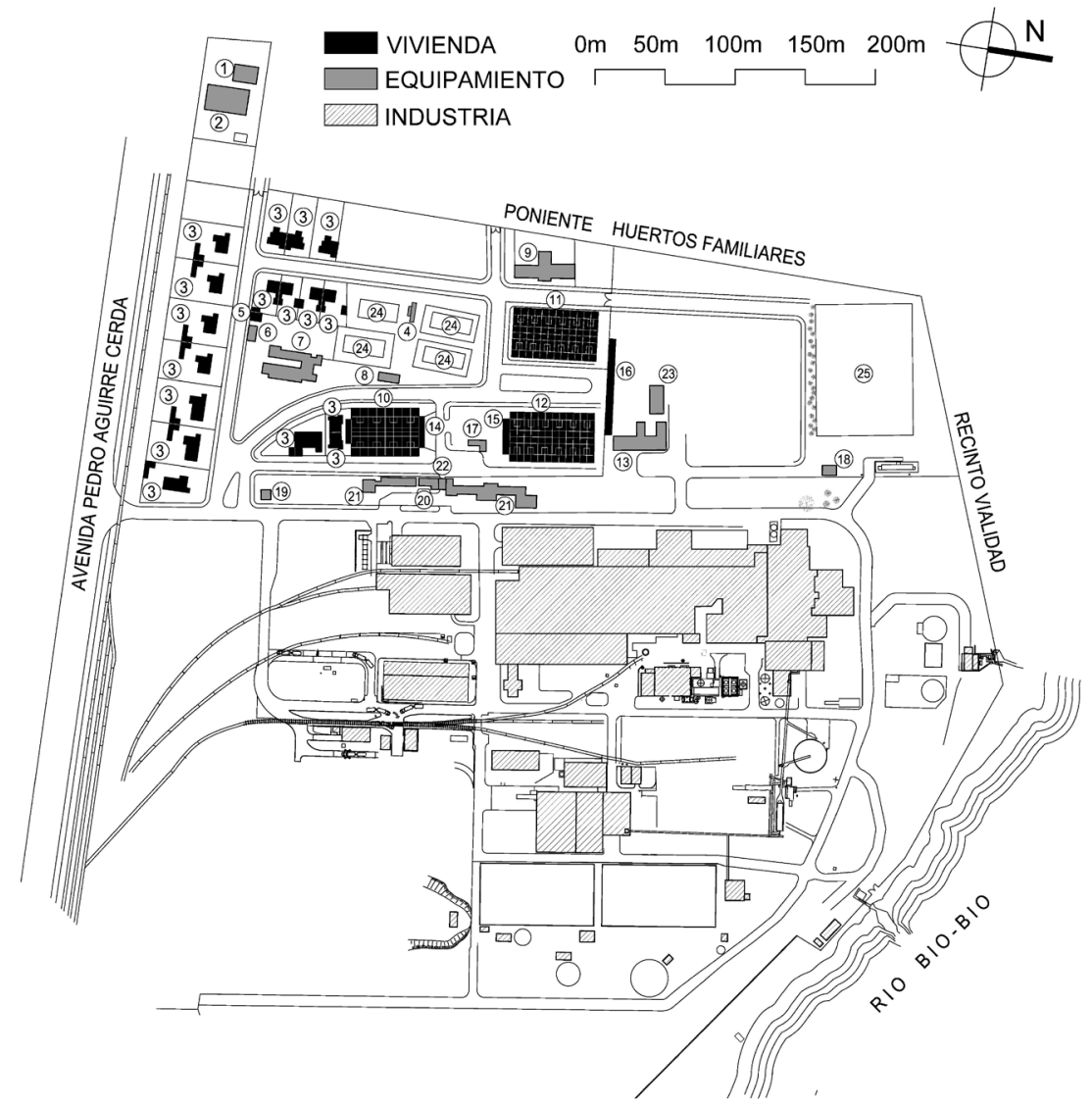
(1) SEDE SINDICAL
(8) KINDER
(15) BOXES BLOCK D
(22) PORTERIA
(2) GIMNASIO
(9) JARDIN INFANTIL
(3) CHALET
(10) BLOCK B
(4) CAMARINES
(11) BLOCK C
(16) BOXES BLOCK CY D
(17) REFUGIO
(23) BODEGA
(5) HABITACION SERVIDUMBRE
(12) BLOCKD
(18) CAMARINES FOOTBALL
(24) CANCHA DE TENIS
(6) SALA DENTAL
(13) OF.RR.II.COMPUTACION (20) POLICLINICO
(7) CASINO
(14) BOXES BLOCK $B$
(21) ADMINISTRACION

El recinto, un polígono industrial controlado, se divide rotundamente entre la fábrica al nororiente y el sector habitacional al norponiente. Se trata de una escisión fundamental que asegura la interdependencia funcional y la necesaria separación ambiental entre dos programas opuestos y, a su vez, mancomunados: vivienda e industria. El sector habitacional, centro de nuestro interés, posee una estructura de sectores bien diferenciados, donde factores funcionales y sociales aparecen bien expresados.

En términos ambientales, es un sistema vecinal ideal permeado por amplias áreas verdes y espacios libres diseñados en franca oposición al complejo programa industrial, donde las edificaciones, no mayores a dos plantas, se supeditan a este concepto urbano (figura 1). Una característica de este sector es la ausencia perceptual de límites particulares, lo que lo identifica como un espacio común y continuo en evidente referencia a la ciudad jardín. A pesar de ello, existen distintas tipologías residenciales que remiten a estratos laborales diferentes, es decir, viviendas para altos cargos, técnicos y operadores. Todas ellas conviven en calles, pasajes y plazuelas, formando un todo continuo y armónico. A este sistema se suman equipamientos y servicios como: jardín infantil, policlínico, sala dental, club, casino y varias áreas deportivas como canchas de tenis, fútbol, etc. Los equipamientos son espacios de encuentro y aglutinación social. De este modo, actualmente es un conjunto donde se intercalan espacios, viviendas y equipamientos, donde se produce una integración espacial que aparenta mitigar las segregaciones sociales. 
Esta vivienda, planificada en la lógica del campamento obrero, privilegió así pabellones con unidades en hilera continua que aseguraban una estandarización social y familiar. Entre ellas no existen divisiones efectivas; los accesos, albergados y enmarcados, uno junto al otro, fomentaban distancias mínimas que aseguraran una homogenización social (imagen 5). El modelo ratifica la idea de un obrero tipo, con familia tipo. De este modo, la vivienda era un engranaje más de la maquinaria productiva que no ameritaba validaciones edilicias relativas a su regularización constructiva y normativa.

En Papeles Bío-Bío, el emplazamiento habitacional es estratégico; las viviendas gerenciales ubicadas próximas al acceso imponen su presencia a modo de control sobre el acceso al conjunto, vigilando quién entra y quién sale. Asimismo, la compartición del espacio público y, en consecuencia, las posibilidades de cruces y encuentros inter-estratos laborales incrementaron el control mutuo. Se trata de una de las formas más básicas del ejercicio efectivo del paternalismo industrial. Para el caso, la relación social entre operarios, técnicos y jefaturas opera con moderación, donde ni la segregación ni la cohesión son fenómenos totales. Si bien las viviendas no están mezcladas en el conjunto, su disposición organizada en diferentes subespacios hace posible su reconocimiento como parte de un mismo espacio habitacional, un recinto semicerrado y autónomo. Para el caso, diferentes mecanismos de congregación estaban dispuestos: equipamientos básicos comunes, infraestructuras, circulaciones, áreas verdes, etc.

En términos tipológicos, se evidencia un lenguaje arquitectónico que deviene tradición. La condición de vivienda aislada, en oposición a la de bloques continuos, señala que para el jefe hay privilegio espacial que opera en aislamiento volumétrico, superficies mayores, rasgos individuales, distancias mayores entre congéneres y exclusividad en el espacio inmediato. Por el contrario, para la vivienda en hilera de los operarios, las superficies mínimas, la repetición estandarizada, la compartición del espacio inmediato y la reducción de distancias de interacción social es determinada como la idónea para su rango. Ambos casos, no obstante, operan sobre un ambiente paisajístico más o menos homogéneo.

No está demás decir que la viviendas pareadas de los mandos medios expresan la reducción de los estándares gerenciales y el incremento de los proletarios, quedando en una situación intermedia en superficies, distanciamientos y áreas verdes inmediatas.

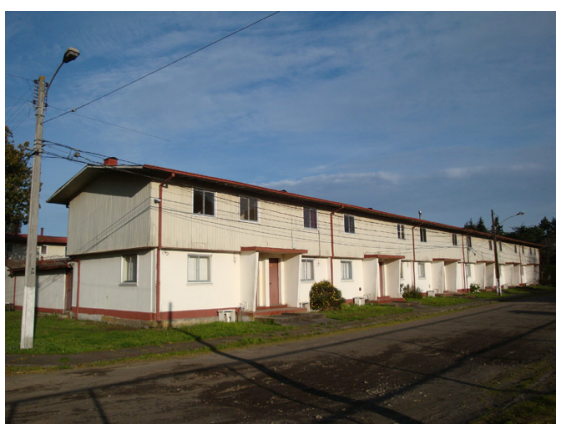

Imagen 4. Pabellón C, colectivos para empleados y operarios solteros (1956.). Arq. F. Urrejola (fuente: Archivo personal Pérez
2016).

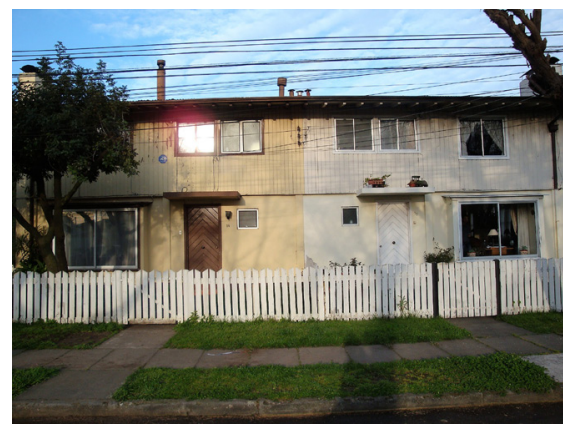

Imagen 3. Viviendas pareadas para mandos medios (fuente: Archivo personal Pérez 2016)

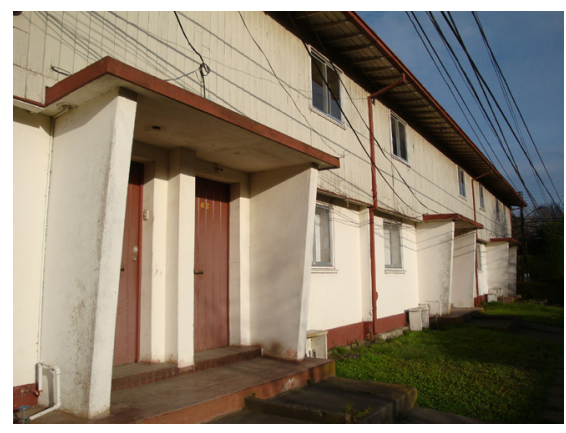

Imagen 5. Acceso a Pabellones colectivos (fuente: Archivo personal Pérez 2016).

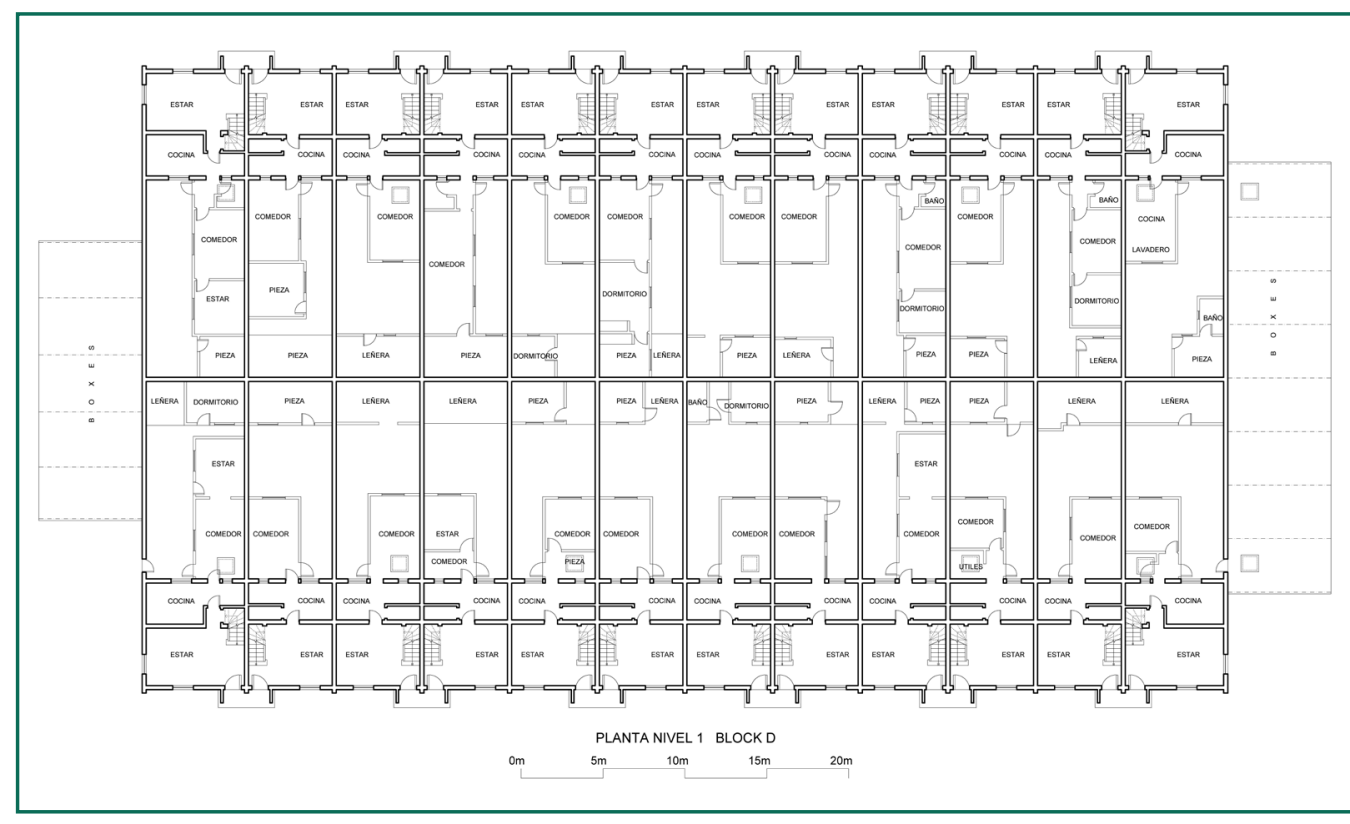

Figura 3. Planta Pabellón D, piso l, colectivos para empleados y operarios solteros (fuente: P. Miranda). 
producción aislado, reglado y apadrinado, donde la empresa favorecía al trabajador a cambio de un comportamiento moderado.

CONCLUSIONES. Estudiar este caso resulta de gran relevancia para el análisis de las relaciones entre espacio residencial y habitar colectivo. El conjunto analizado posee una autonomía primaria, en la medida en que cuenta con infraestructuras mínimas y necesarias que aseguraban, para todas las edades y requerimientos sociales, una vida de barrio. Se trata de una intervención colonizadora del borde sur del Biobío, que asocia la construcción de una industria privada con aseguramiento de la manutención de la fuerza trabajadora en el lugar. La empresa entregaba estas viviendas en comodato, de modo que habitación e industria formaban parte de un ciclo de
En efecto, se trata de un conjunto cuyo control socioespacial admite interacción vecinal, identidad barrial y sentido de pertenencia, renunciando a ciertas

El diseño de tres tipos edificatorios: aislado, pareado y continuo tiene como trasfondo la estratificación política de mediados del siglo XX, a saber, clase baja, media y alta, estigmatización que la industria privada intenta corromper a través de viviendas dignas organizadas entre articulaciones ambientales y funcionales en un espacio homogéneo cerrado, evidentemente dependiente de la industria. Se trata de una solución residencial que hace del conjunto libertades ciudadanas. una pieza propia del engranaje industrial y productivo representativo de un desarrollo económico tutelado y patriarcal. Esta conjunción entre industria y vivienda reafirma un modelo histórico que procura impedir enfrentamientos sociales y, por el contrario, amaina diferencias y contiene los deseos de cambios radicales. Esta construcción espacial, y en consecuencia social, alcanza a mitigar sentimientos de segregación entre los grupos habitacionales que, en el fondo, sin dejar de existir, propician un espacio residencial de una mejor calidad, aparentemente homogéneo, pero sutilmente estratificado. Este resultado ha animado sentimientos de identidad y pertenencia en torno a una empresa privada donde los jefes son el modelo aspiracional visible de los trabajadores

\section{REFERENCIAS}

Acevedo, P., Ciselli, G. y Rojas, C., 2016. "El patrimonio industrial petrolero en la Patagonia: Comodoro Rivadavia (Argentina) y Cerro Sombrero (Chile). Una perspectiva comparada." PASOS Revista de Turismo y Patrimonio Cultural, 14 (4): 981-997.

Alessandri, J., 1969. "Carta del 22 de septiembre de 1969." Recorte, a. III, no 9, s/p.

Aliste, E., Contreras, M. y Sandoval, V., 2012. "Industrialización, desarrollo y ciudad: Transformaciones sociodemográficas y espaciales en la geografía social del Gran Concepción (1950-2010)" INVI 75 (27): 21-71 Disponible en: http://revistainvi.uchile.cl/index.php/INVI/article/view/635

Blog El Paleta, 2005. Don Jorge Alessandri R y la CMPC. Disponible en: http://elpaleta.blogspot.cl/2005/10, don-jorge-alessandri-r-y-la-cmpc.html

Brito, A. y Ganter, R., 2014. "Ciudad obrera: Persistencias y variaciones en las significaciones del espacio. El caso de la siderúrgica Huachipato y su influencia en el desarrollo urbano del Gran Concepción." Revista EURE. 40 (121): 29-53. Disponible en: http://dx.doi.org/10.4067/S0250-71612014000300002

Burotto, C. 1968. "Planeamiento urbano en la Intercomuna de Concepción." Revista AUCA. 3 (13): 59-72. Capron, G. y Esquivel, M. T., 2016. “El enclave urbano, lógica socioespacial de la periferia urbanizada y sus efectos sobre la segregación residencial y la fragmentación urbana." Cuadernos de Geografía: Revista Colombiana de Geografía, 25 (2): 125-149. Disponible en: https://doi.org/10.15446/rcdg.v25n2.54720 Domínquez. P., 2011. Cerro Sombrero, Arquitectura Moderna en Tierra del Fuego. Santiago de Chile: Consejo Nacional de la Cultura y las Artes
Echeñique, A. y Rodriguez, C., 1990. Historia de la Compañía de Aceros del Pacífico S.A. Huachipato consolidación del proceso siderúrgico chileno 1905-1950. Santiago de Chile: CAP S.A. de Inversiones. Fábrica y Conjunto Papeles Bio Bio. Archivo fotográfico, Fotografía B/N.

Fuentes, P., 2006. "Mort de la rue o la introducción de los principios del urbanismo moderno en Chile. Ejercicios de la época." Revista Urbano, 9 (14): 27-33.

Monsálvez. D.: Jaña, P. y Ruiz. P. 2014. "La influencia norteamericana en el proyecto siderúrgico chileno: La Compañía de Acero del Pacifico (CAP) y la usina Huachipato." Universum (Ta/ca), 29 (2): 203-219. Disponible en: https://dx.doi.org/10.4067/S0718-23762014000200014

Papeles Bio Bio, s/f. Nuestra Empresa. Primera planta productora de papel de impresión en Chile. Disponible en: http://www.papelesbiobio.cl/empresa.htm

Pérez, L. y Fuentes, P., 2010. "El Plan Regulador Intercomunal de Concepción de 1963. (PRIC): Inicios de la Planificación Metropolitana." En Pérez, L. e Hidalgo, R. (Coords.), Concepción metropolitano: evolución y desafios. Santiago de Chile: Universidad de Concepción y Pontificia Universidad Católica de Chile, 45-58. Pérez, L., 2016. Registro fotográfico Conjunto papeles Bío Bio, Fotografía color.

Real Academia de la Lengua Española (RAE), 2018a. Enclave. Disponible en: http://dle.rae.es/?id=F74YOPs Real Academia de la Lengua Española (RAE), 2018b. Recinto. Disponible en: http://dle.rae.es/?id=VRPLnt7 Revista Recorte. CMPC, 1967-1980. Revista Recorte, Números 1-42. Concepción, Chile. 